\title{
Ultimate bearing capacity of a pipeline on clayey soils: Slip-line field solution and FEM simulation
}

\author{
Fu-Ping Gao ${ }^{\mathrm{a}, *}$, Ning Wang ${ }^{\mathrm{a}}$, Bo Zhao ${ }^{\mathrm{a}, \mathrm{b}}$ \\ ${ }^{a}$ Key Laboratory for Mechanics in Fluid Solid Coupling Systems, Institute of Mechanics, Chinese Academy of Sciences, Beijing 100190, China \\ ${ }^{\mathrm{b}}$ CNPC Offshore Engineering Company Limited, Beijing 100028, China
}

\section{A R T I C L E I N F O}

\section{Article history:}

Received 9 March 2013

Accepted 3 September 2013

Available online 26 September 2013

\section{Keywords:}

Collapse loads

Slip-line field theory

Plane-strain FEM

Collapse loads

Steel catenary riser

Submarine pipeline

\begin{abstract}
A B S T R A C T
The ultimate bearing capacity of a cylindrical foundation (e.g. a submarine pipeline, circular mooring lines) on clayey soils is analyzed analytically and numerically by employing the slip-line field theory and the plane-strain finite element method respectively. A slip-line field solution is presented, taking into account of circular configuration of the pipe, the pipe embedment, and the pipe-soil interfacial cohesion. The derived bearing capacity factors for a smooth rigid pipe may limit to those for the conventional rectangle-shaped strip footing while the pipe embedment is approaching zero. A plane-strain finite element model is further proposed to simulate the quasi-static process of the pipeline penetrating into the clay soil, in which the contact-pair algorithm and adaptive meshing technique are employed, and the Drucker-Prager constitutive model is used for modeling the soil plasticity. Comparison indicates that the present numerical results match well with the derived slip-line solutions. According to the obtained vertical load-displacement curves, concurrently referring to the plastic strain field and the soil incremental-displacement vector field, the shear failure types and the corresponding collapse loads can be thereby determined for the pipeline foundations on clayey soils.
\end{abstract}

c) 2013 Elsevier Ltd. All rights reserved.

\section{Introduction}

The ultimate bearing capacity of a cylindrical foundation, e.g. a submarine pipeline, circular mooring lines etc., is the pressure causing shear failure of the supporting soil immediately below and adjacent to the foundation. In the recently-issued DNV Recommended Practice (Det Norske Veritas, 2010), the vertical stability of pipelines on and in soils has been specially documented, along with the lateral stability. It is highly desired to efficiently evaluate the bearing capacity of cylindrical foundations. When laid on the seabed, the submarine pipeline settles into the soil with certain embedment under the action of its submerged weight. During the laying process or the operating period, additional vertical loads can also be created at the touchdown zones due to the catenary riser actions (see Fig. 1). The bearing capacity of soft clayey sediments is one of the main geotechnical concerns for the vertical stability of pipelines or mooring lines, especially in deepwater conditions.

Unlike the conventional rectangular strip footing, a cylindrical foundation or pipeline holds a circular cross-section. As such, the effective bearing width of the pipe-soil interface is a function of pipeline embedment; and the existing formulas for the ultimate bearing capacity of conventional footing could not be efficiently

\footnotetext{
* Corresponding author. Tel.: +86 10 82544189; fax: +86 1062561284 .

E-mail address: fpgao@imech.ac.cn (F.-P. Gao).
}

employed for evaluating the ultimate load for the pipeline foundations. A proper determination of the ultimate bearing capacity is crucial for evaluation of the on-bottom stability of submarine pipelines in ocean currents and/or waves (see Murff et al., 1989; Gao et al., 2003, 2012).

The settlement and bearing capacity of the pipeline have received much attention in the past few decades. Conventional bearing capacity theories are mainly for the footings with plane bottom (Chen, 1975). In the theoretical analyses, the soil is absolutely divided into the plastic yield zone and the outer elastic deformation zone. Small et al. (1971) treated the pipeline with certain submerged weight as an equivalent uniform distributed pressure upon a rectangular footing, and proposed empirical formulas for the bearing capacity factors by modifying the solutions for a conventional strip footing. Their treatment obviously could not take into account the effects of the circular section of the pipeline. Karal (1977) applied the upper bound theorems of classical plasticity theory to develop a prediction of pipe penetration, idealizing the pipe as a rigid wedge indenter. The approximation of pipeline with wedge indenter might be reasonable at small embedment but error becomes significant with increasing embedment. Upper and lower bound solutions to penetration of a pipe into cohesive soil were presented by Murff et al. (1989). Finite element method was further adopted by Aubeny et al. (2005) for the plane-strain calculation of collapse loads of the pipeline foundation for the soil profiles with the shear strength varying linearly with depth. 

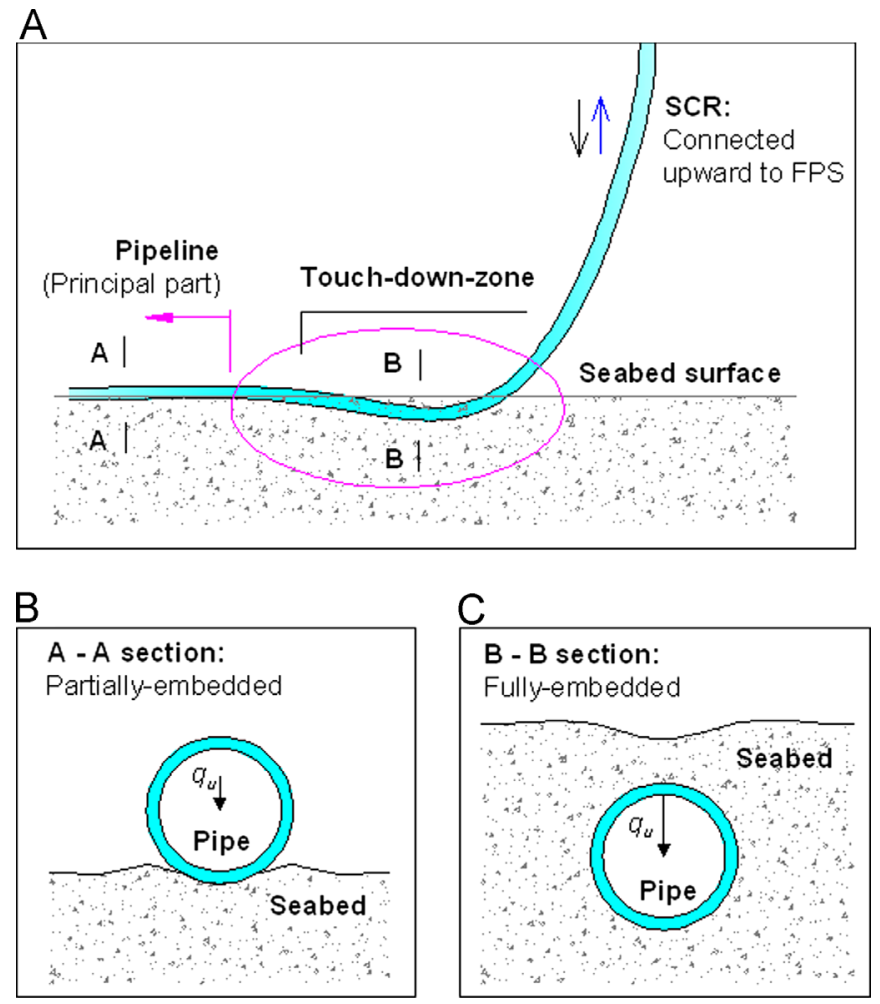

Fig. 1. Sketch map of pipeline embedment in soil.

Hodder and Cassidy (2010) proposed a plasticity model for predicting the undrained behavior of a rigid pipe in clay soils when subjected to the combined vertical and horizontal loading.

Numerical method has also been employed to investigate the bearing capacity of footings, for its potential capability of simulating the development of elasto-plastic deformation beneath the footing at various loading stages. The use of finite element analysis in studying bearing capacity can be tracked back to 1982 when Griffiths (1982) studied the bearing capacity factors of a strip footing (Potts and Zdravkovic, 2001). Pastor et al. (1990) studied the seabed characteristics during pipeline's vertical penetration for both homogeneous and inhomogeneous soils. Bransby et al. (2008) modeled the soil heave around the pipe by using a largestrain finite element formulation, and concluded that the bearing capacity increases slightly with increasing the unit weight of soil, but the effect of surface heave on the bearing capacity is not significant. Due to the special circular cross-section of the submarine pipeline, the quantitative evaluations of ultimate load for pipeline foundations on various sediments and pipe-soil contact conditions are far from being fully achieved.

In this paper, the ultimate collapse loads of the pipeline on Tresca soils are analyzed analytically and numerically by employing the slip-line field theory and the plan-strain finite element method, respectively. Comparisons are made between the slip-line field solutions and the numerical results.

\section{Slip-line field solutions for bearing capacity of cylindrical foundation on clayey soils}

\subsection{Slip-line field for the cylindrical foundations on Tresca soils}

As a typical cylindrical foundation, submarine pipeline's length is usually much larger than its section dimension. As such, the bearing capacity for a submarine pipeline laid upon the horizontally flat seabed may be treated as a plane-strain problem.
In the analytical analysis, the clayey seabed is regarded as a rigid-perfectly plastic material. The Tresca yielding criterion is adopted for the saturated soft clay under undrained conditions. There exists an embedment $\left(e_{0}\right)$ of the pipe with radius of $r$ :

- For the case of $e_{0} / r \leq 1$, the uniform overburden load at the two sides of the pipe $q=0$ and

- for the case of $e_{0} / r>1$, the pipe-soil contact condition can be treated as that for $e_{0} / r=1$, the weight of soil above the pipe center is replaced by an equivalent uniform surcharge pressure $q=\left(e_{0}-r\right) \gamma^{\prime}$, where $\gamma^{\prime}$ is the effective (buoyant) unit weight of soil.

The pipe-soil contact friction is taken into account. Following the assumption by Randolph and Houlsby (1984), the adhesion at the rough pipe-soil interface is taken as a constant factor of the soil cohesion, i.e. $a=\alpha c \quad(0 \leq \alpha \leq 1)$, where $\alpha$ is the pipe-soil interfacial adhesion coefficient, $c$ is the soil shear strength (cohesion). Thus, for a certain point $\mathrm{E}$ at the pipe-soil interface (see Fig. 2), the direction for the slip-line: $\theta_{\mathrm{E}}=\pi / 4-\varphi+\Delta / 2$, in which $\Delta=\arcsin \alpha$. We take $\theta$ as the angle from collapse plane to horizontal plane (clockwise).

According to the well-known slip-line field theory, the coordinates of the slip-lines can be obtained by solving the characteristic functions for slip-lines under certain boundary conditions using the finite-differential method, then the mean stress $\sigma$ (note: $\left.\sigma=\left(\sigma_{1}+\sigma_{3}\right) / 2=\left(\sigma_{x}+\sigma_{y}\right) / 2\right)$ at a certain point in the slip-line field, and the angle $(\theta)$ between the tangent line and the $x$-axis can be calculated from the Hencky stress equations.

As shown in Fig. 2, the boundaries CG and CEB are the Riemann conditions for determining the uniform field CFG and the extrusion filed $C B D$, respectively; the boundaries $C F$ and $C D$ are the regressive Riemann conditions for determining the transition region $\mathrm{CDF}$. Based on the stress analysis, on the line $\mathrm{CG}$, the minimum stress can be determined with the magnitude of $q$ and its direction is vertical. On the line CEB, the maximum stress is located, whose direction is perpendicular to the line CEB for a purely smooth pipe, and whose magnitude is to be determined. Lines CF and CD are the boundary for the filed CFD, whose solution can be determined from the results of the uniform field CFG and those of the extrusion filed CBD. By employing the finitedifferential method, the slip-line fields for the pipeline foundations can be constructed. Fig. 2 gives the slip-line fields for the smooth pipeline $(\alpha=0)$ and the rough pipeline $(\alpha=0.5)$. As indicated in this figure, the whole slip-line field can be divided into three regions, i.e. the uniform region $\mathrm{CFG}$, the extrusion region $\mathrm{CBD}$, and the transition region $\mathrm{CDF}$. The magnitude of the slip-line field for the case of the rough pipelines is larger than that of the smooth pipes.

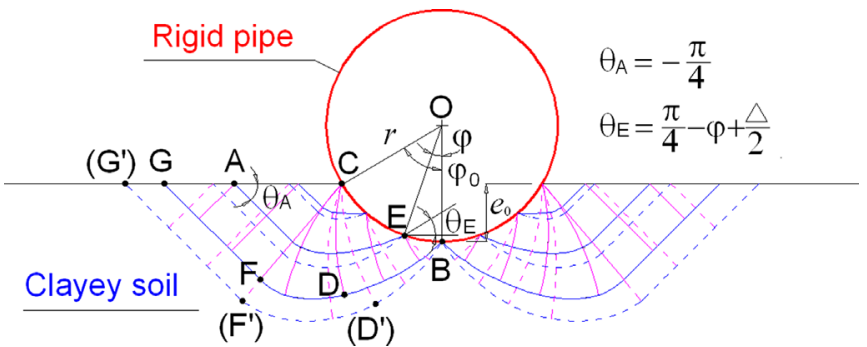

Fig. 2. Slip-line fields of the pipeline foundation on the clayey soil obeying Tresca yield criterion (real lines: smooth pipe $(\alpha=0)$ and dash lines: rough pipe $(\alpha=0.5)$ ). 


\subsection{Collapse loads: slip-line field solutions}

Based on the aforementioned basic assumptions and the constructed slip-line fields, the ultimate load for pipeline foundations can be further derived as follows. The ultimate bearing load $P_{u}$ is expressed in the integral form as

$P_{u}=2 \int_{0}^{\varphi_{0}} r \sigma_{\mathrm{E}, y} d \varphi$

where $\sigma_{\mathrm{E}, y}$ is the vertical component of the pipe-soil contact force; $\varphi_{0}$ is the embedment angle $\angle \mathrm{BOC}$ (see Fig. 2): $\varphi_{0}=\arccos \left(1-e_{0} / r\right.$ ). As shown in Fig. 2, the points $\mathrm{A}$ and $\mathrm{E}$ are along the same $\alpha$ line, and let $\angle \mathrm{BOE}=\varphi$. Submitting the values of $\sigma$ and $\theta$ at points $\mathrm{A}$ and $E$ into the Hencky stress equations:

$\sigma_{\mathrm{A}}-2 c \theta_{\mathrm{A}}=\sigma_{\mathrm{E}}-2 c \theta_{\mathrm{E}}$.

That is

$\sigma_{\mathrm{E}}=\sigma_{\mathrm{A}}+2 \mathrm{c}\left(\theta_{\mathrm{E}}-\theta_{\mathrm{A}}\right)=\sigma_{\mathrm{A}}+2 \mathrm{c}\left(\frac{\pi}{2}+\frac{\Delta}{2}-\varphi\right)$.

Note that $\Delta=\arcsin \alpha$, and $\alpha$ is the pipe-soil interfacial adhesion coefficient. For the direction along the pipe-soil contact arc

$\tau=c \sin \Delta$

$\sigma=\sigma_{\mathrm{A}}+2 c\left(\frac{\pi}{2}+\frac{\Delta}{2}-\varphi\right)+c \cos \Delta$,

in which, $\sigma$ and $\tau$ are the normal and shear stress in the pipe-soil contact plane and $\sigma_{\mathrm{A}}=q+c$. The vertical component of the pipsoil contact force $\sigma_{\mathrm{E}, y}$ can be expressed as

$\sigma_{\mathrm{E}, y}=\tau \sin \varphi+\sigma \cos \varphi$.

That is, the value of $\sigma_{\mathrm{E}, y}$ is the sum of the vertical component of the shear stress $\tau$ and the normal stress $\sigma$ along the pipe-soil contact arc. Compared with the previous assumptions in Gao and Zhao (2012), this updated treatment is much clearer in physics.

Submitting Eqs. (4)-(6) into Eq. (1), the ultimate bearing load $P_{u}$ can be derived as

$$
\begin{aligned}
P_{u} & =2\left[c \sin \Delta\left(1-\cos \varphi_{0}\right)+(q+c+c \cos \Delta) \sin \varphi_{0}\right. \\
& \left.+c(\pi+\Delta) \sin \varphi_{0}-2 c\left(\varphi_{0} \sin \varphi_{0}+\cos \varphi_{0}-1\right)\right] r .
\end{aligned}
$$

Referring to the formula of the bearing capacity for conventional strip footings, the bearing capacity for pipeline foundations may be expressed in the following form:

$\frac{P_{u}}{2 r \sin \varphi_{0}}=c N_{c}+q N_{q}$,

where " $2 r \sin \varphi_{0}$ " is the width of the pipe-soil interface.

Submitting Eq. (7) into Eq. (8), the bearing capacity factor for cohesion $\left(N_{c}\right)$ and the bearing capacity factor for distributed load $\left(N_{q}\right)$ can thereby be obtained

$N_{c}=\frac{\sin \Delta\left(1-\cos \varphi_{0}\right)-2\left(\cos \varphi_{0}-1\right)}{\sin \varphi_{0}}+1+\Delta+\pi+\cos \Delta-2 \varphi_{0}$,

$N_{q}=1$

\subsection{Comparison of bearing capacity between pipeline and conventional strip footings}

In the analysis on the general shear failure mechanism of a conventional rectangular strip footing, e.g. Prandtl-Reissner solution (see Knappett and Craig, 2012), the smooth strip footing carries a uniform pressure on the surface of a mass of homogeneous, isotropic soil; the shear strength parameters for the soil are $c$ and $\phi$; and a surcharge pressure $q$ acting on the soil surface has been taken into account. The following exact solution has been widely used for the ultimate bearing capacity of a rectangular strip footing on the surface of a weightless soil

$\frac{P_{u}}{b}=c N_{c}+q N_{q}$ (for a rectangular strip footing),

where $b$ is the width of the conventional strip footing (note that, for the pipeline foundation, $b=2 r \sin \varphi_{0}$ (see Fig. 2)), $N_{c}$ and $N_{q}$ are the bearing capacity factors, i.e.

$N_{c}=\left(N_{q}-1\right) \cot \phi$,

$N_{q}=e^{\pi \tan \varphi} \tan ^{2}\left(\frac{\pi}{4}+\frac{\phi}{2}\right)$,

in which $\phi$ is the internal angle of soils. For the rectangular strip footing on a pure cohesive soil (i.e. $\phi=0$ ), the bearing capacity factors are $N_{c}=\pi+2$ and $N_{q}=1$.

For the case of the partially-embedded horizontal cylindrical foundation (e.g., a submarine pipeline) on Tresca soils, if the pipeline surface is fully-smooth $(\Delta=0)$, then the bearing capacity factors (9a) and (9b) are simplified as

$N_{c}=(\pi+2)+2\left(\frac{1-\cos \phi_{0}}{\sin \phi_{0}}-\phi_{0}\right)$,

$N_{q}=1$.

Now to examine the two extrema of $N_{c}$ (see Eq. (12a))

$\lim _{\varphi_{0} \rightarrow 0} N_{c}=2+\pi$,

$\lim _{\varphi_{0} \rightarrow \pi / 2} N_{c}=4$

Fig. 3 gives the variation of $N_{c}$ with $e_{0} / r$ for smooth pipes. When $\varphi_{0} \rightarrow 0$ (i.e. the pipeline just touches the soil surface $e_{0} / r=0$ ), the bearing capacity factor $N_{c}$ for pipeline foundations (see Eq. (13a)) matches that for the conventional strip footings. This indicates that, while the pipeline embedment approaching zero, the formulae for the bearing capacity of pipeline foundations degenerate into those for the conventional rectangular-shaped strip footings.

With the increase of the pipeline embedment, the value of $N_{c}$ decreases gradually and finally reaches 4.0 when the pipeline is half buried (see Fig. 3). Therefore, if pipeline foundations are directly simplified as conventional strip footings, the bearing capacity factor $N_{c}$ would be over evaluated, whose error may be up to $28.5 \%$.

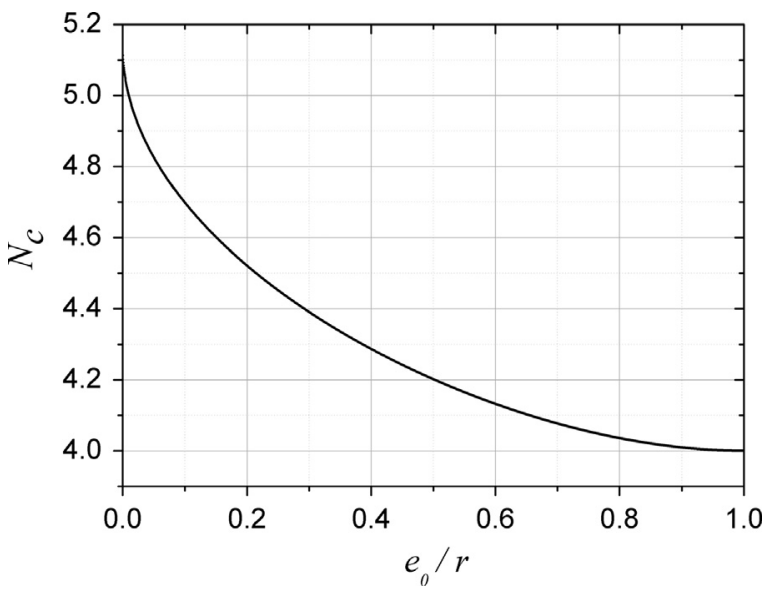

Fig. 3. Variation of $N_{c}$ with $e_{0} / r$ for smooth pipes. 


\subsection{Parametric study on bearing capacity factors}

Based on the derived formulae for the bearing capacity of the partially embedded pipeline on Tresca soils, i.e. Eq. (8), the relationship between the bearing capacity factors $\left(N_{c}\right.$ and $\left.N_{q}\right)$ and the non-dimensional pipeline embedment $\left(e_{0} / r\right)$, and the pipe-soil interfacial cohesion coefficient $(\alpha)$ can be established.

Fig. 4 gives the variation of $N_{c}$ with the parameters $e_{0} / r$ and $\alpha$. As shown in Fig. 4, when $\alpha>0.2$, the values of $N_{c}$ initially decreases to a minimum value, then increases continuously with the increase of $\alpha$; when $\alpha<0.2$, the values of $N_{c}$ decreases with increasing $\alpha$. The effect of $\alpha$ on $N_{c}$ gets more significant with the increase of pipeline embedment $\left(e_{0} / r\right)$. The maximum value of $N_{c}$ emerges $\left(N_{c}=5.71\right)$ under the condition of fully-bonding $(\alpha=1)$ and non-burial $\left(e_{0} / r=0\right)$.

For better understanding the bearing capacity of pipeline foundations, the dimensionless ultimate bearing load $P_{u} / \mathrm{cr}$ is introduced. Eq. (7) is thereby rewritten as

$P_{u} / c r=2\left(N_{c}+\frac{q}{c} N_{q}\right) \sin \varphi_{0}$,

where the bearing capacity factors $N_{c}$ and $N_{q}$ are calculated with Eq. (8). Fig. 5 gives the variation of $P_{u} / c r$ with the dimensionless pipeline embedment $\left(e_{0} / r\right)$ and the pipe-soil interfacial cohesion coefficient $(\alpha)$, under the condition that the embedment is less than the pipeline radius $(q=0)$. For the fixed value of $\alpha, P_{u} / c r$ increases with increasing $e_{0} / r$. For the fixed values of $e_{0} / r, P_{u} / c r$ increases with increasing $\alpha$; the effects of $\alpha$ on $P_{u} / c r$ are higher for

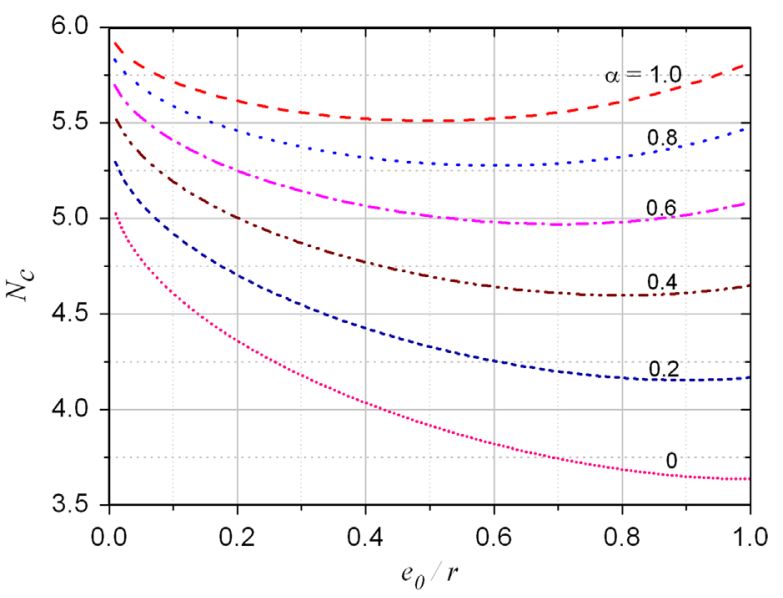

Fig. 4. Variation of $N_{c}$ with $e_{0} / r$ for various values of $\alpha$.

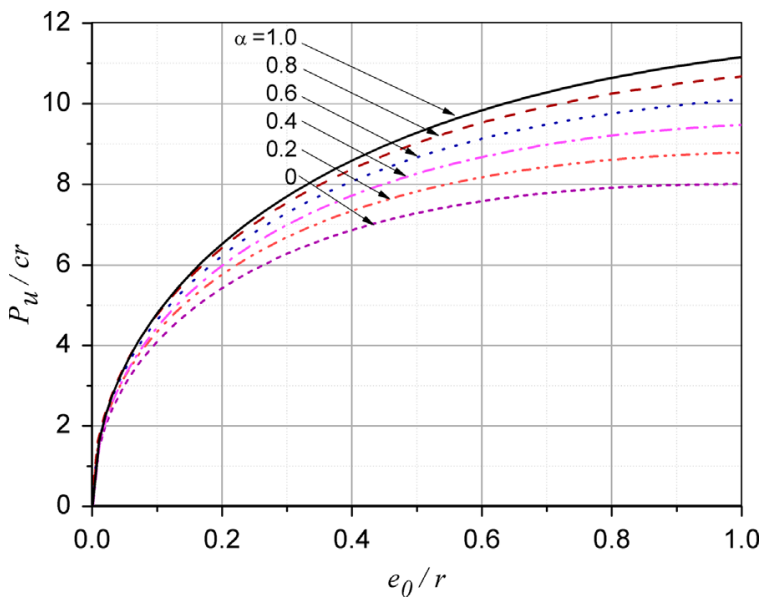

Fig. 5. Variation of $P_{u} / c r$ with $e_{0} / r$ for various values of $\alpha(q=0)$. larger values of $e_{0} / r$. When $\alpha=1$ and $e_{0} / r=1, P_{u} / c r$ reaches its maximum value.

\section{Plane-strain FEM simulations}

\subsection{FE model for pipe penetrating into soils}

Similar with the assumptions adopted in the aforementioned slip-line filed analysis, in the finite element (FE) analysis, the pipeline is also regarded as a rigid cylinder for the reason that the stiffness of steel pipeline is usually much larger than that of soils. In the FE model, the clay soil is simulated with the linear DruckerPrager (D-P) elastoplasticity constitutive model. As well-known, the clayey soil can be essentially assumed to behavior as an elastic Tresca material, if undrained bearing capacity is considered, and as an elastic $c-\phi$ material (e.g. Mohr-Coulomb or D-P material), if drained bearing capacity is under investigation (also see Potts and Zdravkovic, 2001).

The FEA mesh of the plane-strain model and the boundary conditions are illustrated in Fig. 6. The vertically loaded surface footing resting on a soil is usually treated as a symmetric planestrain problem (see Potts and Zdravkovic, 2001). As such, half of the full geometry needs to be considered with the following boundary conditions:

(1) Symmetry Boundary: the displacement in the $x$ direction and the rotation in the $x-y$ plane are restrained, i.e. only the vertical displacement (settlement) is permitted.

(2) Right Boundary: the displacement in the $x$ direction and the rotation in the $x-y$ plane are restrained.

(3) Bottom Boundary: a fixed boundary, i.e. the displacements in both $x$ and $y$ directions and the rotation in the $x-y$ plane are restrained.

(4) Interface between Pipe and Seabed Surface: the contact-pair algorithm is adopted to simulate the pipe-soil interaction.

(5) The left non-contact surface of the seabed is treated as a free boundary.

Note that the pipe-soil interface changes while the pipe penetrating into the soil. As such, it is crucial to deal with the contact surface between seabed and pipeline in the numerical

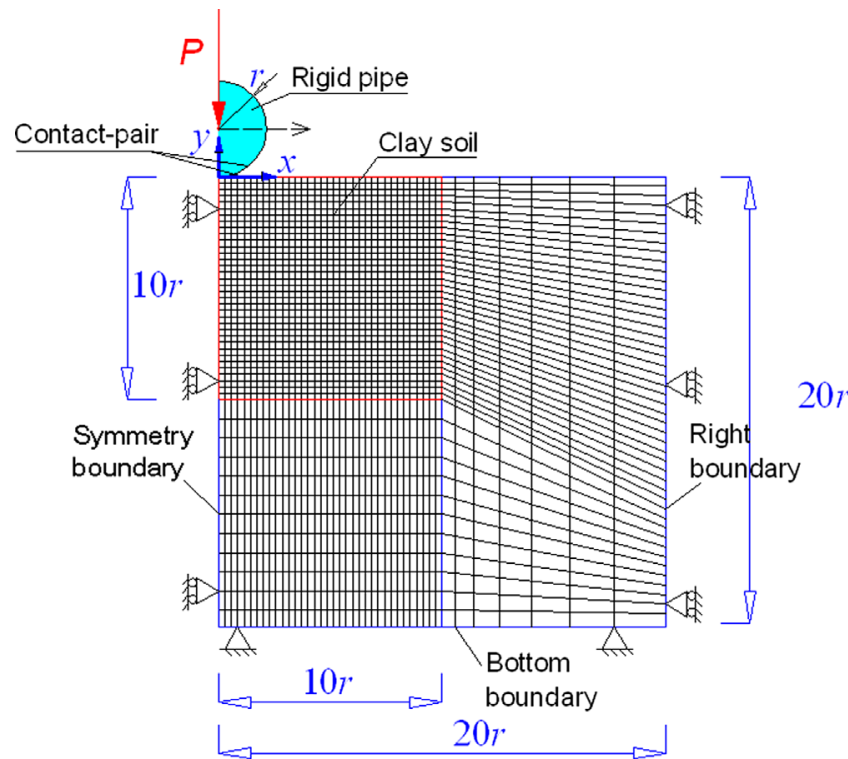

Fig. 6. Illustration of the FEM mesh of plane-strain model and the boundary conditions. 
modeling. The contact-pair algorithm provided in the ABAQUS software (Hibbitt, Karlsson and Sorensen, 2006) is adopted, and the pipe-soil friction is defined by the Penalty Function with the advantage that it guarantees the positive definiteness of sparse matrix in the calculation. To avoid large distortion of FEM elements causing the calculation misconvergence, self-adaptive mesh technology is employed in the numerical modeling.

To obtain high calculation efficiency, the finite element mesh gets more refined at closer proximity to the pipe. Based on the results of a series of trial calculations, the width of numerical model is set as $20 r$ and the depth as $20 r$ ( $r$ is the pipe radius).

The linear Drucker-Prager model is chosen for its simplicity to simulate the elastoplastic behavior of the clay soils, which provides for a possibly noncircular yield surface in the deviatoric plane ( $\pi$-plane) to match different yield values in triaxial tension and compression, associated inelastic flow in the deviatoric plane, and separate dilation and friction angles. The linear DruckerPrager criterion, i.e. the yield surfaces in the meridional $p-t$ plane, is written as (see Hibbitt, Karlsson and Sorensen, 2006)

$F_{s}=t-p \tan \beta-d=0$,

where $t$ is the deviatoric stress measure, i.e.

$t=\frac{1}{2} q\left[1+\frac{1}{K}-\left(1-\frac{1}{K}\right)\left(\frac{R}{q}\right)^{3}\right]$,

in which $p$ is the equivalent pressure stress; $K$ is the ratio of the yield stress in triaxial tension to the yield stress in triaxial compression and, thus, controls the dependence of the yield surface on the value of the intermediate principal stress (when $K=1.0, t=q$ implying that the yield surface is the von Mises circle in the deviatoric principal stress plane), $R$ is the third invariant of deviatoric stress, $q$ is the Mises equivalent stress; $\beta$ is the slope of the linear yield surface in the $p-t$ stress plane and is commonly referred to as the friction angle of the material; and $d$ is the intercept of the linear yield surface in the $p-t$ stress plane and is commonly referred to as the cohesion of the material.

The values of friction angle $(\phi)$ and cohesion $(c)$ for the MohrCoulomb model can be provided directly with the available triaxial experimental data. For such a plane-strain problem and for the case of associated plastic flow, the values of the parameters in the linear Drucker-Prager model are related to the Mohr-Coulomb parameters by the following relationships (see Hibbitt, Karlsson and Sorensen, 2006)

$\tan \beta=\frac{\sqrt{3} \sin \phi}{\sqrt{1+(1 / 3) \sin ^{2} \phi}}$,

$\frac{d}{c}=\frac{\sqrt{3} \cos \phi}{\sqrt{1+(1 / 3) \sin ^{2} \phi}}$.

In the simulations, the parameters of soil are chosen as follows: elasticity modulus $E=1.0 \mathrm{MPa}$, mass density $\rho=1.8 \times 10^{3} \mathrm{~kg} / \mathrm{m}^{3}$, Poisson ratio $v=0.3$. Other soil parameters (e.g. $c$ and $\phi$ ) are various for the parametric study in Section 3. As aforementioned, the pipe is treated as a rigid cylinder with the radius $r=0.25 \mathrm{~m}$. The pipe-soil friction coefficients are varied $\alpha=0-0.7$.

\subsection{Numerical results and analyses}

The pipe settlements and soil plastic deformations under the action of the submerged weight of the pipe are simulated with the proposed numerical model. The curves for the vertical loaddisplacement relationships are plotted to estimate the failure type of pipe foundation and the corresponding bearing capacity. The development of plastic zone underlying the pipe under vertical loads is examined numerically. Furthermore, comparisons are made between the present numerical results and the slip-lines theoretical solutions.

\subsubsection{Failure types of pipe foundation and determination of collapse loads}

For a conventional rectangular strip footing under vertical loading, there are usually three failure types, i.e. the general shear failure, the local shear failure and the punching shear failure. The failure type can be determined relatively easily with the loaddisplacement curve. Nevertheless, the situation may become more complex for the pipeline foundations with circular section.

The curves for pipeline vertical load-displacement relationships and the soil plastic strain distributions at the critical value along the load-displacement curve are shown in Fig. 7(a) and (b) and (c), respectively, for two typical values of cohesion of clayey soils. Fig. 7(a) indicates that, those two vertical loaddisplacement curves are similar in profile with that of conventional strip footing suffering from a punching shear failure. Fig. 7(b) gives the distribution of soil equivalent plastic strain at critical value of $W_{s}$ for the soils with $\phi=0$ and $c=20.21 \mathrm{kPa}$, which shows that the plastic zone is located just beneath the pipe, i.e. no plastic deformation occurs besides the pipeline. This plastic deformation distribution indicates that the pipe foundation $(\phi=0$ and $c=20.21 \mathrm{kPa}$ ) suffers a punching shear failure. The corresponding ultimate load (or named as "collapse load") $P_{u} \approx 38.0 \mathrm{kN} / \mathrm{m}$ (see Fig. 7(a)). Unlike the plastic deformation in Fig. 7(b), the plastic zone in Fig. 7(c) extends from beneath the pipe to the seabed surface besides the pipe, indicating the pipe foundation ( $\phi=0$ and $c=5.77 \mathrm{kPa}$ ) suffers a general shear failure (the ultimate load $P_{u} \approx 10.0 \mathrm{kN} / m$, see Fig. $\left.7(\mathrm{a})\right)$.

Therefore, it is usually difficult to determine the failure type of pipeline foundations only from the profile of vertical load-displacement curve, as their profiles are similar for different failure types due to the special circular sections of the pipe. When the turning point (from gentle to steep incline) along the loaddisplacement curve is not distinguishable, the plastic zone beneath the pipe foundation should be referred for determination of the ultimate loads.

The development of plastic zone beneath the pipe foundation and the corresponding incremental-displacement vector will be discussed in the following sub-sections.

\subsubsection{Development of soil plastic zone beneath the pipeline}

The development of plastic zone beneath the pipe foundation with the increase of vertical loads is shown in Fig. 8. The soil parameters $\phi=0$ and $c=5.77 \mathrm{kPa}$ and the pipe-soil friction coefficient $\alpha=0.7$. When the effective downward load on the pipe is small, the plastic zone is just located beneath the pipe (see Fig. 8(a)). With the increase of the effective loads, the plastic zone extends gradually to the soil surface beside the pipe (see Fig. 8(b) and (c)), indicating the pipeline foundation suffers a general shear failure. Compared with the smooth pipe (the ultimate load $P_{u} \approx 10.0 \mathrm{kN} / \mathrm{m}$ for $\alpha=0$, see Fig. 7(c)), this rough pipe obtains a higher ultimate loads due to the increase of pipe-soil friction coefficient $\left(P_{u} \approx 15.1 \mathrm{kN} / m\right.$ for $\alpha=0.7$, see Fig. $8(\mathrm{c})$ ).

Fig. 9 shows the development of the punching shear failure to the pipe foundation $(\phi=0$ and $c=20.21 \mathrm{kPa})$. As shown in Fig. 9 (a)-(c), with increasing effective loads to pipe, the range of soil plastic zone becomes deeper beneath the pipe, i.e. in this case, no plastic deformation extending to the soil surfaces beside the pipe. For such a bigger value of soil cohesion, the punching shear failure of pipe foundation is more apt to be triggered. 
a

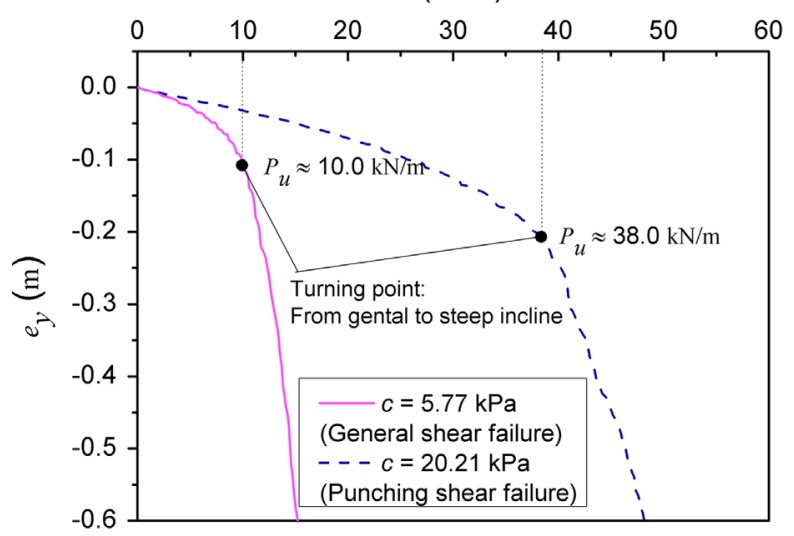

b

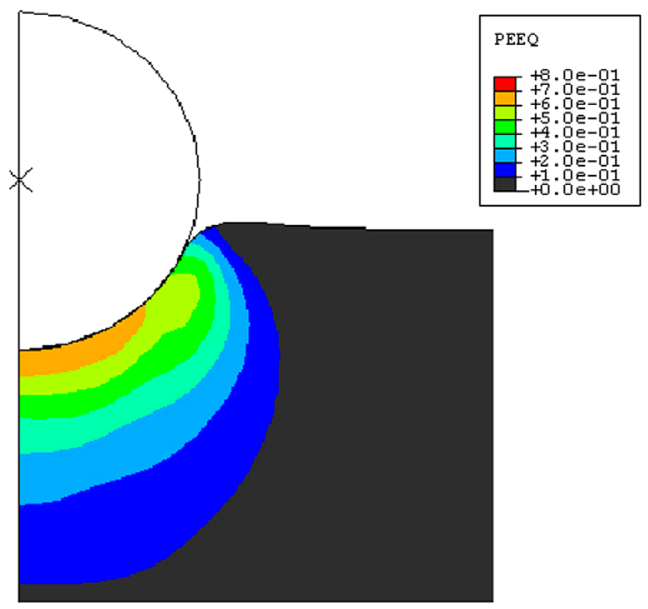

C

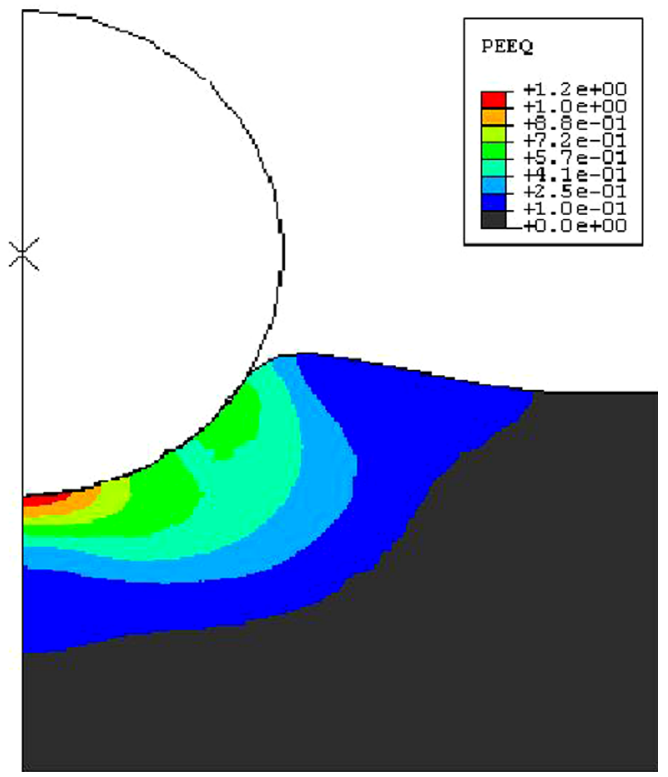

Fig. 7. (a) Vertical load-displacement curves for the pipeline foundations on clay soils $(r=0.25 \mathrm{~m}$ and $\mu=0)$; and the corresponding equivalent plastic zones; (b) punching shear failure ( $\phi=0$ and $c=20.21 \mathrm{kPa}$ ); and (c) general shear failure ( $\phi=0$ and $c=5.77 \mathrm{kPa}$ ).

\subsubsection{Incremental-displacement vector field beneath the pipeline}

Fig. 10 shows the incremental-displacement vector fields at the ultimate load for the pipe foundation suffering a general shear failure for various pipe-soil friction coefficients. The orientation of the vectors indicates the direction of movement and their length the magnitude of movement. As it is the orientation of the vectors and their relative magnitude that indicates the failure mechanism (see Potts and Zdravkovic, 2001), the absolute magnitude of the incremental displacements is irrelevant and no magnitude scale is given in the figures.

It is indicated in Fig. 10 that, pipe-soil friction coefficient $(\alpha)$ has some influence on the range of incremental-displacement vector field. The failure mechanism for the rough pipe is deeper and wider than that for the smooth pipe. For the rough pipe ( $\alpha=0.5$ ), the soil just adjacent to the bottom of the pipe trends to move downward together with the settlement of the pipe. However, for the smooth pipe ( $\alpha=0)$, the adjacent soil is pressed by the moving pipe to spread aside, which induces the obvious upheaval of the soil surface in the proximity of the pipe. With the increase of $\alpha$, the range of incremental-displacement vector field increases, which finally brings the increase of ultimate load to the pipe foundation.

Similar numerical results are also obtained for the pipe foundation with the increase of the internal friction angle from $\phi=0^{\circ}$ to $18^{\circ}$, as shown in Fig. 11. Comparison between Figs. 10 and 11 indicates that the increase of internal friction angle of soil further enlarges the range of incremental-displacement vector field.

\subsection{Comparison with slip-line field solutions}

Slip-line field solutions for the ultimate load of submarine pipelines on a purely cohesive soil obeying Tresca yield criterion have been obtained (see Section 2.). According to the slip-line field theory, the coordinates of the slip-lines were obtained by solving the characteristic functions for slip-lines under boundary conditions using the finite-differential method, as shown in Fig. 2. The slip-line fields of theoretical solutions are quite comparable with the profiles of incremental-displacement vector fields at ultimate load when the pipe foundation suffers a general shear failure for various pipe-soil friction coefficients (see Figs. 10 and 11).

To make a quantitative comparison with the above solutions of slip-line theory, parametric studies are carried out. The parameters and the numerical results of the ultimate loads for pipe foundations are listed in Table 1. Note: in the Table 1, the symbol * presents the numerical results for pipe foundations suffering punching shear failure; the examined pipes are purely smooth $(\alpha=0)$.

The aforementioned finite element method (see Section 3.1) is adopted for analysis on the determination of bearing capacity. That is, the failure type of pipeline foundations and the corresponding ultimate loads are determined by not only the profile of vertical load-displacement curve, but also by referring to the development of the plastic zone beneath the pipe, especially when the turning point from gentle to steep-incline along the load-displacement curve is not distinguishable.

Fig. 12 gives comparison between the present FEA results with the solutions of slip-line filed theory for smooth pipes $(\alpha=0)$, indicating their results match well with same trends of the variation of $P_{u} / c r$ with $e_{0} / r$, i.e. the dimensionless ultimate loads $\left(P_{u} / \mathrm{cr}\right)$ increase gradually with the increase of the dimensionless pipe embedment $\left(e_{0} / r\right)$.

\section{Concluding remarks}

The ultimate bearing capacity of a cylindrical foundation or pipeline on purely-cohesive clay soils is analyzed analytically and numerically by employing the slip-line field theory and the planstrain finite element method, respectively. Parametric studies are 
a

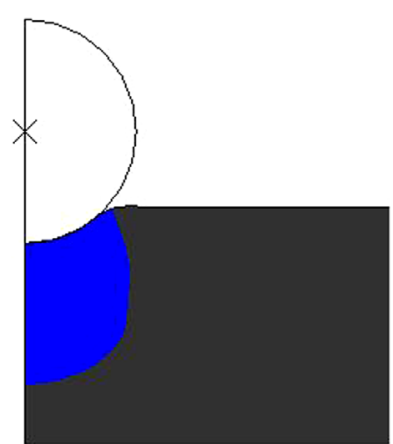

b

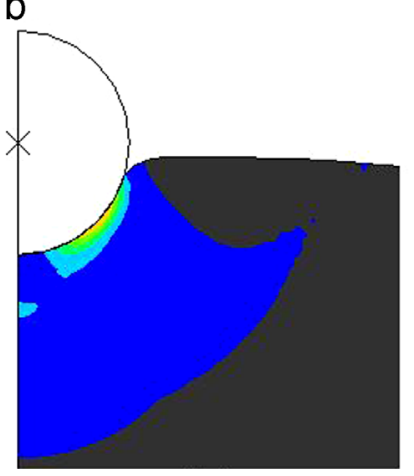

C

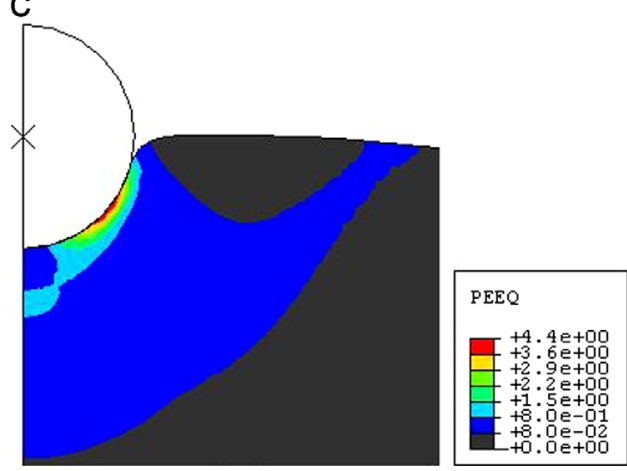

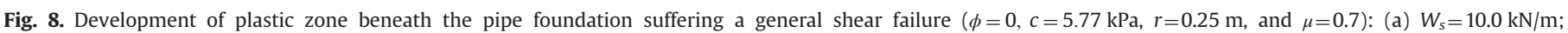
(b) $W_{s}=14.7 \mathrm{kN} / \mathrm{m}$; and (c) $W_{s}\left(=P_{u}\right)=15.1 \mathrm{kN} / \mathrm{m}$.

a

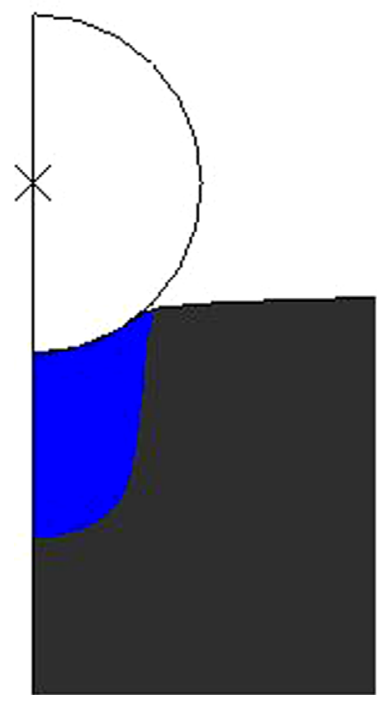

b

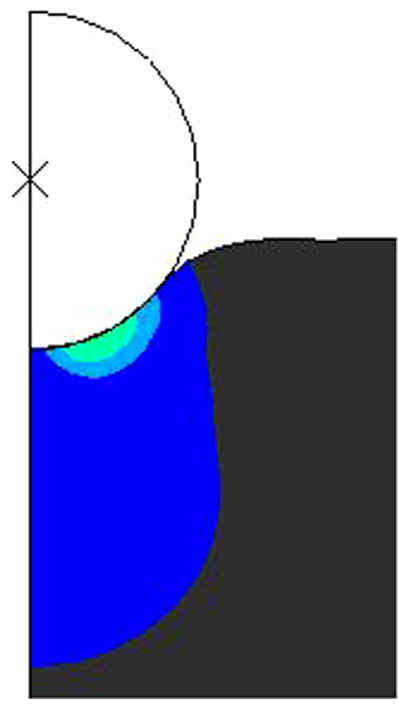

C

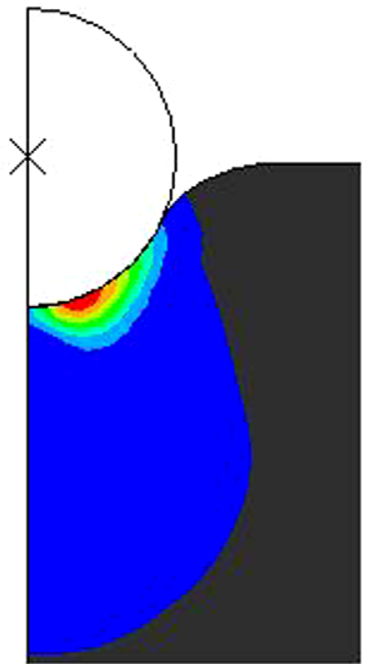

PEEQ

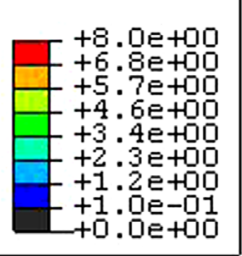

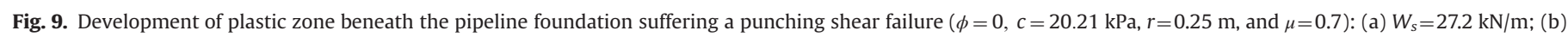
$W_{s}=38.0 \mathrm{kN} / \mathrm{m}$; and (c) $W_{s}=45.0 \mathrm{kN} / \mathrm{m}$.

a

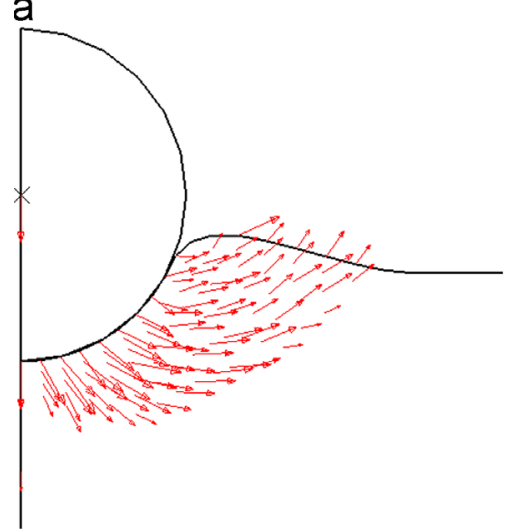

b

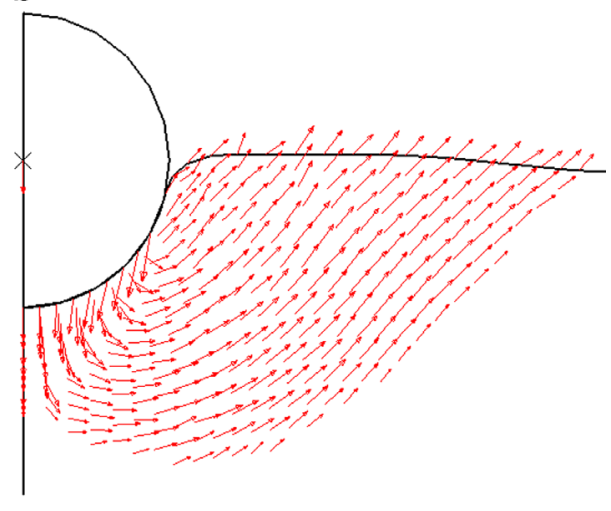

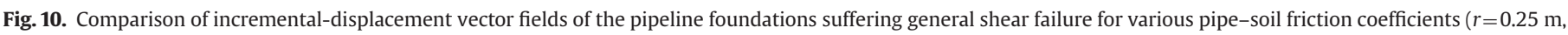
$\phi=0, c=5.77 \mathrm{kPa}$ ): (a) $\alpha=0$ and (b) $\alpha=0.5$.

performed on the bearing capacity factors and the dimensionless ultimate load. The following conclusions can be drawn:

(1) Due to the special circular arc shape of the cylindrical foundations, the constructed slip-line fields for the partially-embedded pipeline on Tresca soils are different from those of the conventional strip footings. The pipe-soil contact friction also has much effect on the magnitude of the slip-line fields. The slip-line field for the rough pipeline is larger than that for the smooth pipeline.

(2) The bearing capacity factors for cylindrical foundations, i.e. $N_{c}$ and $N_{q}$, are derived. When the embedment of a smooth pipeline approaches zero, the bearing capacity factors degenerate into those for the conventional strip-line footing (Prandtl's mechanism). With the increase of the pipeline embedment, the bearing 

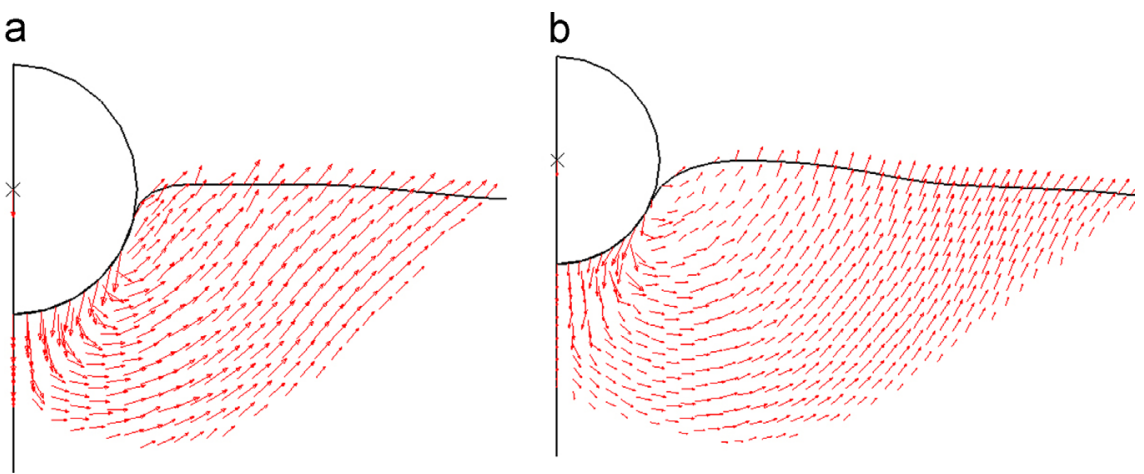

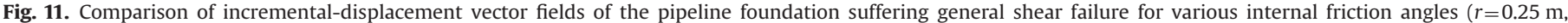
$c=5.77 \mathrm{kPa}$, and $\alpha=0.5$ ): (a) $\phi=0$ and (b) $\phi=18^{\circ}$.

Table 1

Numerical results of bearing capacity for pipe foundations.

\begin{tabular}{llccc}
\hline$e_{0}(\mathrm{~m})$ & $e_{0} / r$ & $c(\mathrm{kPa})$ & $P_{u}(\mathrm{kN} / \mathrm{m})$ & $P_{u} / \mathrm{cr}$ \\
\hline 0.08 & 0.336 & 1.15 & 2.0 & 6.93 \\
0.11 & 0.444 & 5.77 & 10.0 & 6.93 \\
0.15 & 0.596 & 10.39 & 19.3 & 7.43 \\
0.19 & 0.784 & 15.01 & 29.5 & 7.86 \\
0.20 & 0.808 & 20.20 & $38.0^{*}$ & $\mathrm{NA}$ \\
0.23 & 0.920 & 28.86 & $55.0^{*}$ & $\mathrm{NA}$ \\
\hline
\end{tabular}

Note: symbol * presents the numerical results for pipe foundations suffering punching shear failure.

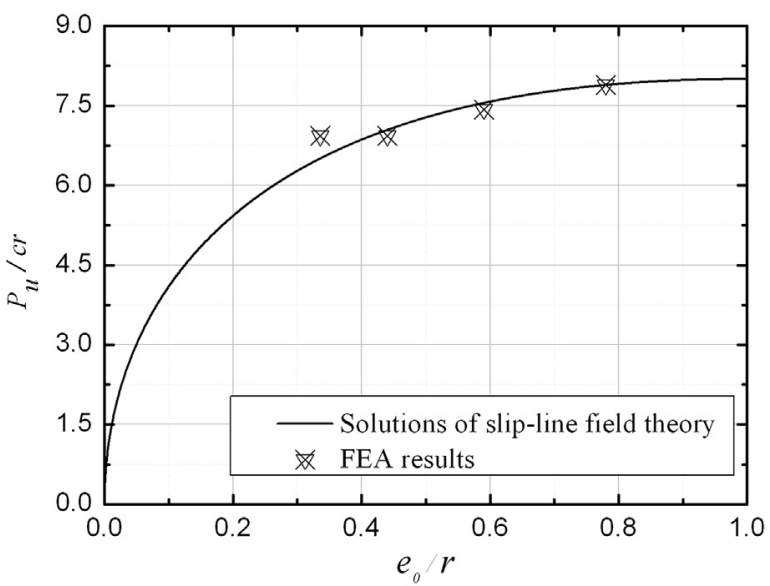

Fig. 12. Variation of $P_{u} / c r$ with $e_{0} / r$ : comparison between present FEA results with the solutions of the slip-line filed theory.

capacity factor $N_{c}$ decreases gradually, and finally reaches the minimum value (4.0) when the embedment equals to pipeline radius. As such, if pipeline foundations are directly simplified as conventional strip footings, the bearing capacity factor $N_{c}$ would be over evaluated.

(3) The bearing capacity factors for pipeline foundations are significantly influenced by non-dimensional pipeline embedment and pipe-soil frictional coefficient.

(4) A dimensionless ultimate bearing load $P_{u} / \mathrm{cr}$ is introduced. Under the condition that the embedment is less than pipeline radius, $P_{u} / c r$ increases with increasing pipeline embedment $\left(e_{0} / r\right)$ for the fixed value of the pipe-soil frictional coefficient $(\alpha) ; P_{u} / c r$ increases with increasing the pipe-soil frictional coefficient $(\alpha)$ for the fixed value of $e_{0} / r$. The effects of $\alpha$ on $P_{u} / c r$ get higher for larger values of $e_{0} / r$.
(5) To simulate the quasi-static process of a cylindrical foundation/pipeline penetrating into the soil, a plane-strain finite element model is proposed for analysis of the bearing capacity of pipeline on clayey soils, in which the adaptive grid technique and "contact pair" arithmetic are employed.

(6) The development of soil plastic zone and the incrementaldisplacement vector field beneath the pipeline are examined numerically. According to the obtained pipeline vertical loaddisplacement curves, concurrently referring to the plastic strain field and/or the soil incremental-displacement vector field, the shear failure type (e.g., general shear failure and punching shear failure) and the collapse loads can be thereby determined. The present numerical results match well with the analytical solutions of slip-line theory in plasticity mechanics.

Note: in this study, the vertical penetration/bearing capacity of a pipeline on a clayey soil is analyzed, in which the effect of hydrodynamic loading from waves and currents is not taken into account. This effect on the on-bottom stability of a submarine pipeline in the marine environment is a key issue for the pipeline design practice.

\section{Acknowledgments}

This work was financially supported by the National Natural Science Foundation of China (Grant nos. 11372319 and 11232012) and the Major State Basic Research Development Program of China (973 Program) (Grant no. 2014CB046204).

\section{Appendix A. Slip-line field theory}

The slip-line field theory is on the basis of equilibrium equations and the failure criterion of the material. In this study, the soil is assumed to obey Tresca failure criterion, i.e.

$\left(\sigma_{x}-\sigma_{y}\right)^{2}+4 \tau_{x y}^{2}=4 c^{2}$

Let $\sigma=\left(\sigma_{1}+\sigma_{3}\right) / 2=\left(\sigma_{x}+\sigma_{y}\right) / 2$ and refer to the Mohr circle, then

$\sigma_{x}=\sigma-c \sin 2 \theta$

$\tau_{x y}=c \cos 2 \theta$

$\sigma_{y}=\sigma+c \sin 2 \theta$

For a plane strain problem regarding, the equilibrium equations are

$\frac{\partial \sigma_{x}}{\partial x}+\frac{\partial \tau_{x y}}{\partial y}=0$ 
$\frac{\partial \tau_{x y}}{\partial x}+\frac{\partial \sigma_{y}}{\partial y}=0$

(A.3b)

Submitting Eq. (A.2a)-(A.2c) to Eq. (A.(3a) and A.3b), the characteristic functions for slip-lines can be derived as

$\frac{d y}{d x}=\operatorname{tg} \theta$ (for $\alpha$ line)

$\frac{d y}{d x}=-\operatorname{ctg} \theta$ (for $\beta$ line)

and the Hencky stress equations as

$\sigma-2 c \theta=$ const 1 (along $\alpha$ line)

$\sigma+2 c \theta=\operatorname{const} 2$ (along $\beta$ line)

where $x$ and $y$ are the coordinates for the point in the slip-line field, $\sigma$ is the mean stress at a certain point in the slip-line field, $\theta$ is the angle between the $\alpha$ tangent line and the $x$-axis, $c$ is the cohesion of soil. When the boundary conditions are given, the stress and the corresponding slip-lines can be determined.

\section{References}

Aubeny, C.P., Shi, H., Murff, J.D., 2005. Collapse loads for a cylinder embedded in trench in cohesive soil. International Journal of Geomechanics 10, 320-325.

Bransby, M., Zajac, P., Amman, S., 2008. Finite element analysis of the vertica penetration of "on-bottom" pipelines in clay. In: Proceedings of the 18th International Offshore and Polar Engineering Conference, pp. 245-249.
Chen, W.F., 1975. Limit Analysis and Soil Plasticity. Elsevier Scientific Publishing Co., New York.

Det Norske Veritas, 2010. On-bottom stability design of submarine pipelines. Recommended Practice, DNV-RP-F109.

Gao, F.P., Gu, X.Y., Jeng, D.S., 2003. Physical modeling of untrenched submarine pipeline instability. Ocean Engineering 30, 1283-1304.

Gao, F.P., Han, X.T., Cao, J., Sha, Y., Cui, J.S., 2012. Submarine pipeline lateral instability on a sloping sandy seabed. Ocean Engineering 50, 44-52.

Gao, F.P., Zhao, B., 2012. Slip-line field solution for ultimate bearing capacity of a pipeline on clayey soils. Theoretical \& Applied Mechanics Letters 2, 051004.

Griffiths, D.V., 1982. Computation of bearing capacity factors using finite elements. Géotechnique 3, 195-202.

Hibbitt, Karlsson, Sorensen, 2006. ABAQUS Theory Manual, Version 6.5-1.

Hodder, M.S., Cassidy, M.J., 2010. A plasticity model for predicting the vertical and lateral behaviour of pipelines in clay soils. Géotechnique 60 (4), 247-263.

Karal, K., 1977. Lateral stability of submarine pipelines. In: Proceedings of the Annual Offshore Technology Conference, Houston TX, Paper OTC-2967, pp. 7178.

Knappett, J.A., Craig, R.R.F., 2012. Craig's Soil Mechanics. Taylor \& Francis, London. Murff, J.D., Wagner, D.A., Randolph, M.F., 1989. Pipe penetration in cohesive soil. Géotechnique 39 (2), 213-229.

Pastor, J., Turgeman, S., Avallet, C., 1990. Predicting the phenomenon of burying through gravity in purely cohesive sedimentary sea beds. Géotechnique 39 (4), 625-639.

Potts, D.M., Zdravkovic, L., 2001. Finite Element Analysis in Geotechnical Engineering: Application. Thomas Telford Ltd., London.

Randolph, M.F., Houlsby, G.T., 1984. The limiting pressure on a circular pile loaded laterally in cohesive soil. Géotechnique 34, 613-623.

Small, S.W., Tambuvello, R.D., Piaseckyj, P.J., 1971. Submarine pipeline support by marine sediment. In: Proceedings of the Annual Offshore Technology Conference, Houston TX, Paper OTC-1357, pp. 309-318. 